Original Research

\title{
Acupuncture in Palliative Care
}

Franco Cracolici *, Simonetta Bernardini, Rosaria Ferreri

Local Health Unit South-East Tuscany, Department of Integrated Medicine, Pitigliano Hospital, Tuscany Region, Italy; E-Mails: francocracolici@yahoo.it; bernardini@s-bernardini.it; tyvvf@tin.it

* Correspondence: Franco Cracolici; E-Mail: francocracolici@yahoo.it

Academic Editors: Elio Rossi, Sonia Baccetti and Mariella Di Stefano

Special Issue: Experience and activities of the Regional Tuscan Centre for Integrative Medicine

OBM Integrative and Complementary Medicine 2019, volume 4, issue 1

doi:10.21926/obm.icm.1901006
Received: October 14, 2018

Accepted: January 22, 2019

Published: January 31, 2019

\begin{abstract}
:
Background: Acupuncture is an innovative and scientifically supported treatment for oncologic patients, as well as an effective palliative care option. At the Palliative Care Department of "Misericordia" Grosseto Hospital in Tuscany, real integration of acupuncture in palliative medicine has been possible. The objective of this work is to retrospectively evaluate patient treatment outcomes obtained using an integrated medical care approach administered within a palliative care unit.
\end{abstract}

Methods: Medical records of oncology patients admitted to the palliative care unit who voluntarily underwent integrated therapy with acupuncture were retrospectively analyzed.

Treatment was innovative and personalized and included the use of

- points based on Traditional Chinese Medicine (TCM),

- points based on the Microsystems Acupuncture Technique,

- points based on psychic action (Shen Ling).

Codified evaluation indexes were used to rate patient status at the beginning of treatment and after 1-2 months. Treatment outcomes were evaluated for oncologic patients voluntarily participating as palliative care outpatients or inpatients receiving integrated acupuncture therapy.

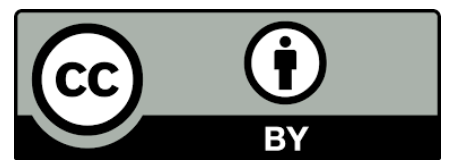

(C) 2019 by the author. This is an open access article distributed under the conditions of the Creative Commons by Attribution License, which permits unrestricted use, distribution, and reproduction in any medium or format, provided the original work is correctly cited. 
Results: 172 cancer patients treated over a two-year period received a total of approximately 600 treatments, with $92.4 \%$ of patients reporting improvement of symptoms. Examination of patient data obtained from the SF 12 questionnaire and of patient assessments based on the Edmonton Symptoms Assessment Scale (ESAS) revealed remarkable improvement in perceived health 2 months after initiation of integrated therapy $(p<0.01)$. Specifically, marked post-treatment improvement in symptoms of pain, fatigue, nausea, sleep disturbance, anxiety, loss of appetite, shortness of breath, cough and wellbeing were observed, with no improvement observed for dry mouth or depression. No major side effects were reported.

Conclusions: Acupuncture is a promising and safe adjunctive therapy for management of common symptoms that afflict oncological patients during all stages of disease, including symptoms of patients nearing the end of life in a home or hospice setting.

\section{Keywords}

Acupuncture; palliative care; quality of life; integrative medicine

\section{Introduction}

Palliative care is an "approach that improves the quality of life (QoL) of patients and their families who are faced with the problems associated with incurable diseases."(Based on the 2002 World Health Organization (WHO) definition "Palliative Care approach improves quality of life of patients and families who manage problems related to incurable diseases, by prevention and suffering relief through early identification, exhaustive evaluation and pain treatment and other physical, psycho-social, and spiritual problems.) This field of treatment is progressively gaining attention within an overall public debate regarding the importance of ensuring that terminally ill patients receive care that preserves their dignity and provides relief from suffering at the end of life [1, 2].

In this context, palliative medicine is not intended to "cure" disease but aims to "cure" the person. This goal is accomplished by caring for both the patient and the patient's family by administering treatment to first relieve pain and other distressing symptoms followed by treatment to soothe anxiety and fears related to the concept of death [3]. For the best treatment outcome, it is essential to develop a support system that incorporates a multidisciplinary team that is able to address each and every need of the patient and the patient's family [4]. From a historical perspective, Traditional Chinese Medicine and acupuncture have greatly contributed to palliative care and complex cancer patient treatments by employing minimally invasive procedures with very low incidences of adverse effects [5] and harmful pharmacological interactions. Such treatments have exhibited great effectiveness in improving QoL from both a physical and a psychological standpoint.

Acupuncture has been used in China and other parts of the world for thousands of years and is defined as the insertion of small, thin, sterile, solid stainless steel needles into specific points on the body to treat or prevent symptoms [6]. The use of this discipline in both oncology and in 
palliative care is scientifically supported and is employed by world-renowned hospitals in the US, including Memorial Sloan-Kettering Cancer Center, MD Anderson Cancer Center and Dana Farber Cancer Institute [7]. According to the latest studies, acupuncture effects in oncological applications are ascribable to systemic and biochemical reactions and bioelectrical modifications that act to decrease release of inflammatory cytokines [8], reduce T-cell activation [9], increase adenosine release [10] and alter neuropeptide-based activation, opioid peptide-based activation, peptidic hormone activation or stem-cell activation [11].

Emerging evidence suggests that acupuncture should be considered for management of cancer treatment symptoms and side effects that include pain [12-14], fatigue [15, 16], hot flashes $[17$, 18], anxiety, depression [19] and other symptoms [20]. Moreover, strong evidence is accumulating to support its use for treatment of nausea and vomiting [21]. Meanwhile, according to Meidell [22] who conducted a Swedish hospice study, acupuncture improved xerostomia in most elderly patients [23-25].

Indeed, the impact of acupuncture on patient QoL can be remarkable. An interesting report published in 2010 by Dean Clower at the Dana Farber Cancer Institute shows that acupuncture improves QoL and terminal symptoms, such as anxiety, fatigue, pain and depression, when used as palliative therapy in patients with advanced cancer [26]. Specifically, QoL scores in the report reflected improvements in pain severity, pain interference, physical and psychological distress, life satisfaction, and mood states during treatment, with a sustained benefit observed at 12 weeks.

Meanwhile, the positive impact of acupuncture on mood and sleep has also been documented [27]. Lau's recent and important systematic review and meta-analysis [28] of the effectiveness of acupuncture and related therapies for symptom management during palliative cancer care concluded that "acupuncture and related therapies are effective in reducing pain, fatigue, and in improving QoL when compared with conventional intervention alone among cancer patients."

Finally, National Comprehensive Cancer Network (NCCN $\left.{ }^{\circledR}\right)$ guidelines pertaining to cancer pain in adults recommend using acupuncture as part of a broad series of integrated pharmacological interventions to address pain issues. Notably, the guidelines emphasize the importance of using integrated treatment regimens for treating the weakest patients, such as elderly, pediatric and terminal oncologic patients who often tolerate standard pharmacological treatments poorly.

Because acupuncture is definitely an innovative option to treat cancer and provide effective palliative care, it is the most studied and scientifically supported non-standard care option. Indeed, accumulating evidence suggests that an optimal treatment strategy for cancer and palliative care patients likely would integrate various complementary disciplines into a customized treatment program optimized for each patient. At the Center of Lenitherapy in Tuscany's "Roberto Ciabatti" Misericordia Grosseto Hospital (South East Local Health Unit), real integration of acupuncture, phytotherapy and homeopathy disciplines into palliative medicine has beeit is the name dedicated to this departmentn achieved [29]. Since the inception of this integrative program in 2015, it has been guided by the Tuscan health model to create a public hospital ward where patients could receive conventional and complementary therapies either at the same time or with greater overall integration [30]. This program differs from other European or American patient treatment programs, whereby alternative therapies are mainly restricted to the clinic and conventional therapies to the hospital ward, with an absence of cooperation between clinic and hospital 
doctors.

At the Misericordia Grosseto Hospital, the oncology department fosters a multidisciplinary environment based on a model of cooperation between doctors skilled in integrated medicine and other hospital staff. Within this model system, joint visits by caregivers to diverse palliative care patients (outpatients, day patients, hospital/hospice inpatients) and their families are conducted in order to improve QoL and optimize treatment outcomes, survivorship and end-of-life care. Using an evidence-based approach, recommendations are made for personalized care based on integration of conventional and nonconventional therapies.

The objective of the study is to assess the impact of real integration of acupuncture and standard treatment on patient QoL and treatment outcomes for an unselected population of cancer patients, including patients with advanced disease or those nearing the end of life. While randomized controlled trials (RCTs) are the gold standard for establishing treatment effectiveness, patient-reported outcomes can demonstrate whether patients perceive acupuncture to be effective. Therefore, in this study we conduct a preliminary evaluation of integrated treatment outcomes. This study also paves the way for a future in-depth prospective study that will assess the overall effectiveness of integrated treatment on the patient's family and on healthcare staff as indirect effects of cancer patient treatment.

\section{Materials and Methods}

In this work, the medical records of cancer patients who underwent integrated treatment incorporating acupuncture from December 2015 through December 2017 were retrospectively analyzed. Only patients who voluntarily chose to undergo acupuncture treatment after preselection by a health care provider were included. The possibility of undergoing treatment was also offered to patients' families and to healthcare staff, but data from medical records of these study participants is not included in the scope of this study.

All treatments were provided in the patient's room or in the clinic by licensed and experienced (8 years minimum) staff acupuncturists and consisted of weekly sessions aimed toward improving QoL, with particular attention to:

-physical suffering,

-gastric and bowel disease,

-bone and muscular pain,

-emotional grief,

-will to live,

-fear of death.

For each patient, an innovative and personalized treatment was used. Acupuncture treatment included use of the following:

- points based on Traditional Chinese Medicine (TCM) [31-36],

- points based on Microsystems Acupuncture Technique,

- points based on a psychic action (Shen Ling) [37-40].

The Microsystems Acupuncture Technique consisted of auricular, abdominal and navel, wristankle, hand and Su-Jok acupuncture procedures, as well as Chinese Scalp Acupuncture and Yamamoto New Scalp Acupuncture (YNSA). Treatment modalities included the following details: 
-needles were positioned on the surface without manipulation,

-needles were infixed usually for no more than 15 minutes,

-for excessively weak patients, Seirin's new Pyonex needle (also known as a "flea pin") were used instead of conventional needles to stimulate a point without causing any painful sensation,

- in this first phase of the project, moxibustion was not used to stimulate the vital points (even if it is expected to be used in the future).

Codified evaluation indexes (Edmonton Symptoms Assessment Scale (ESAS) and SF 12 questionnaire) were used at treatment initiation and again after 2 months of integrated therapy. Using the ESAS scale, symptoms are rated on the scale of 0 to 10, with 10 signifying "worst possible." Symptoms included in the modified ESAS are pain, fatigue, nausea, depression, sleep disturbance, anxiety, loss of appetite, shortness of breath and general well-being. A change of 1 point or greater is considered clinically significant [41].

The SF 12 scale (widely used scale for the assessment of general health and QoL) is comprised of an online score calculator used to analyze data that measure characteristics reflecting the two dimensions of health for generation of a physical component summary (PCS) and a mental component summary (MCS). Each summary is based on a scale of 0 to 100 , whereby a lower score reflects greater disability and a higher score reflects lower disability. We also collected data on cough and dry mouth using a progressive scale of 0 to 10.

Categorical variables were reported as absolute and percentage values. Continuous variables were reported as mean \pm SD. Comparisons between two groups were performed using the student's $t$-test for unpaired data, with a value of $p<0.05$ considered significant. Statistical calculations were performed using SPSS version 21 (SPSS Statistics Inc., La Jolla, CA, USA).

\section{Results}

During the first two years of the project, the Palliative Care Department of the Hospital of Grosseto performed more than 900 acupuncture sessions for approximately 320 outpatients and their families, 45 residents of the hospice facility and 8 palliative care medical professionals.

For the analysis, only data of the subgroup of cancer patients (at any disease stage) were included: 172 patients were treated during the two-year study and they received a total of about 600 treatments. It is noteworthy that 54 patients received treatment during the first year of disease activity, with the remaining two-thirds of patients treated during the second year of the program. Demographic information for the patient cohort is shown in Table 1. The population was predominantly composed of women. The overall patient mean age was 74 years and the age range was 32-89 years. The most commonly treated symptoms were cancer pain and complex chronic pain syndromes. Other frequently treated disorders were nausea, vomiting, stomatitis/mucositis, asthenia/fatigue and anxiety/stress/panic crisis. Intensity of symptoms was coded using the ESAS and changes in QoL were measured from data collected from completed SF 12 questionnaires (Table 2). The most commonly used traditional acupuncture points were $43 \mathrm{BL}, 6 \mathrm{CV}, 4 \mathrm{CV}, 17 \mathrm{CV}$, $4 \mathrm{GV}, 23 \mathrm{BL}, 36 \mathrm{ST}, 12 \mathrm{CV}$ and $11 \mathrm{LI}$. Each patient received an average of 3 treatment sessions (range 1-6).

Considering only patients affected by neoplastic pathology (excluding data of patient family members and hospital staff), after 2 months of integrated therapy $92.4 \%(n=159)$ of patients 
reported some improvement in symptoms. Examination of items addressed in the SF 12 questionnaire or of patients assessed using the ESAS revealed remarkable improvement in perceived health 2 months after the start of integrated therapy $(p<0.01)$ and statistically significant improvement $(p<0.05)$ for all treated symptoms (Pain, Fatigue, Nausea, Sleep disturbance, Anxiety, Loss of appetite, Shortness of breath, Cough, Well-being), but no improvement of dry mouth and depression. No major side effects and no clinical complications were reported, except for small localized hematomas at acupuncture needle insertion sites and 3 cases of mild post-treatment headache.

Table 1 Demographic characteristics of the population.

\begin{tabular}{ll}
\hline Parameter & Patients (n=172) \\
\hline Age (range) & $74(32-89)$ \\
Male sex (\%) & $77(45 \%)$ \\
Hospice Residents (\%) & $17(10 \%)$ \\
Type of Cancer (n) & \\
Breast (\%) & $45(26 \%)$ \\
Colonrectal (\%) & $48(28 \%)$ \\
Lung (\%) & $33(19 \%)$ \\
Gynecological (\%) & $14(8 \%)$ \\
Pancreas (\%) & $21(12 \%)$ \\
Cholangiocarcinoma & $3(2 \%)$ \\
Head-Neck (\%) & $5(3 \%)$ \\
Prostate (\%) & $3(2 \%)$ \\
\hline
\end{tabular}

Table 2 Intensity of symptoms coded according to Edmonton Symptoms Assessment Scale (ESAS) and changes in quality of life according to the SF 12 questionnaire before and after treatment.

\begin{tabular}{lccc}
\hline & $\begin{array}{c}\text { Before } \\
(\text { Mean } \pm \text { SD })\end{array}$ & $\begin{array}{c}\text { After } \\
(\text { Mean } \pm \text { SD })\end{array}$ & $p$ \\
\hline Pain & $4,2 \pm 3,8$ & $2 \pm 1,7$ & $<0,05$ \\
Fatigue & $5,2 \pm 4,1$ & $3 \pm 2,1$ & $<0,01$ \\
Nausea & $2,8 \pm 3,2$ & $1,8 \pm 2,4$ & $<0,05$ \\
Depression & $2,8 \pm 3,1$ & $2,5 \pm 3,5$ & n.s \\
Sleep disturbance & $6.2 \pm 2.6$ & $4.9 \pm 2.7$ & $<0,01$ \\
Anxiety & $5 \pm 3,6$ & $2,8 \pm 3$ & $<0,01$
\end{tabular}




\begin{tabular}{lccc} 
Loss of appetite & $1,6 \pm 2,3$ & $1,4 \pm 2,2$ & $<0,05$ \\
Shortness of breath & $4,9 \pm 3,1$ & $3,9 \pm 2$ & $<0,05$ \\
Well-being & $4,7 \pm 1,3$ & $7,8 \pm 1,3$ & $<0.01$ \\
SF 12 PCS & $39,62 \pm 22,27$ & $76,19 \pm 19,29$ & $<0,01$ \\
SF 12 MCS & $58,68 \pm 20,46$ & $79,85 \pm 14,59$ & $<0,01$ \\
Cough & $4,2 \pm 3,2$ & $3,6 \pm 2,1$ & $<0,05$ \\
Dry Mouth & $3,8 \pm 3,2$ & $3,5 \pm 3,3$ & n.s. \\
\hline
\end{tabular}

SF 12 PCS: Physical Component Summary; SF 12 MCS: Mental Component Summary.

\section{Discussion}

Over the last 10 years interest in integrated oncology has increased considerably and a substantial body of evidence now exists to support acupuncture use in this treatment field. Although very few researchers have evaluated acupuncture use in an inpatient setting, in 2017 Garcia et al. reported great treatment efficacy for acupuncture applied in a clinical setting [42]. In the retrospective study described here, acupuncture treatment is evaluated for use in conjunction with standard medical oncological treatment of a heterogeneous and unselected patient population. The current study provides more complete data that demonstrate the benefits of acupuncture, warranting more detailed clinical trials and prospective studies that will ultimately guide future clinical research.

From an operational standpoint, in our study $96 \%$ of patients who accepted acupuncture treatment in the first year of the program requested duplication of acupuncture sessions in the second year. This result suggests high patient acceptance of this type of therapy and highlights a great need for patient-centered treatment design. Although evidence from scientific studies indicates that patients who most frequently use acupuncture are women of high social status between 30 and 45 years of age [43], these data are not confirmed in our study. This discrepancy may stem from the fact that the study population was selected for pathology and underwent acupuncture sessions on the advice of health care providers. For each patient an average of only 2 to 4 sessions of acupuncture were performed, indirectly demonstrating the speed of therapeutic efficacy and reflecting a high level of patient approval. Although well-designed RCTs are needed to demonstrate acupuncture efficacy before its incorporation as part of standard cancer care, we observed no major secondary side effects and no patient refused to continue treatment after the first session. However, regardless of demographic data, this study indicates that for cancer patients not selected based on disease severity, acupuncture can achieve significant improvement of many often disabling symptoms that affect treatment adherence (Pain, Fatigue, Nausea, Sleep disturbance, Anxiety, Loss of appetite, Shortness of breath, Cough and Well-being). Based on informal feedback from patients, caregivers and hospital personnel, acupuncture care delivery was practicable and well received. In addition, it appears that the involvement of caregivers and patients' family members undoubtedly improved patient adherence to and acceptance of treatment.

Finally, regardless of the numerical data, it is important to emphasize that the aspect of joint efforts between physicians with integrated medical skills and other hospital staff facilitated the 
creation of a care network that contributed most to the success of this innovative experience. While data derived from the application of acupuncture in real clinical practice can be used to guide clinical decision-making and achieving optimal outcomes, several limitations of this study should be noted:

1. It is not a randomized controlled clinical trial and therefore the specific efficacy of acupuncture needling cannot be determined.

2. As this was a retrospective study of clinical data collected in an unselected population, it is possible that other clinical factors (disease stage, treatment, time since diagnosis) and history of other medical interventions administered for symptom management between the initial and follow-up visit could be responsible for measured improvements.

3. Ratings from patients collected by the acupuncture physician may have possibly and inadvertently influenced patients to provide more favorable ratings.

4. The possibility of a placebo effect must be considered, since patients willing to receive acupuncture services on the advice of caregivers may be a unique, self-selected group with higher expectations for a positive result. For example, Mao and colleagues [43] found that breast cancer patients who were willing to enroll in an acupuncture trial had higher response expectations than those who would decline participation.

5. The choice of points has not been completely standardized and those we used partially diverged from points most frequently described in cancer pathology and relief reports published from 1936-2014 (namely ST36, LI4, PC6, SP6, ST21, CV12, CV17 and TE6). Because our selected protocol was intended to allow for customization of treatment, this factor likely introduced a statistical bias in our results. However, in this first phase of project implementation we preferred to implement the procedure in this way in order to address each individual patient's unique psychic aspects. This element appeared fundamentally important to us after consideration of the study population of end-of-life patients, family members and health workers subjected to very strong emotional situations.

6. While here we have assessed the effect of treatment only on the oncological patient population, we should also consider that the involvement of family members and caregivers may have indirectly influenced measurable efficacy and increased patient appreciation of and predisposition to treatment. Moreover, acupuncture may have prevented burnout of palliative care workers by alleviating their own stress and anxiety (data not shown). Nevertheless, in our opinion the possibility of treatment offered to relatives of patients and health workers is the most innovative aspect of this program. From our point of view, treatment should not be limited to the care of psychophysical aspects of the patient, but should also be extended to others sharing the context in which the patient lives, works and receives care. By promoting an alliance among all caregivers (within the family and in the medical care setting), a virtuous circle is created whereby patients and their families never feel abandoned and receive concrete support at both psychophysical and care management levels. It is also important to consider that the prevalence of burnout among health personnel who relate daily with cancer patients and end-of-life issues is very high and we think it is appropriate that they also have access to therapy that offers psychological and emotional support.

Despite these limitations, the data analyzed during the first two years of work are encouraging. 
As far as retrospective data are concerned, the results undoubtedly show that our treatment strategy had positive effects on patients and should therefore be valued and continued. For future study development, these data will be evaluated prospectively to pave the way for studies using larger patient samples. In addition, the study design may be extended to allow for random comparisons of integrated and standard treatments for patient groups subdivided according to cancer severity and type.

With an adequate sample, it would also be useful to extrapolate data collected from the subgroup of patients in the terminal phase. In fact, we believe that acupuncture can be a valid tool to promote a scientifically integrated medical vision that serves to treat people by addressing both psychophysical and environmental factors during their care. In this vision, palliative medicine, more than any other discipline, should be built upon an articulated therapist network to care for the patient and address suffering, as articulated in a quote by A. Malliani, "When the system doesn't work anymore here is the terminal patient...to die gracefully is the highest life message that a person can testify." [44]

\section{Conclusions}

In clinical practice, acupuncture appears to be an important and safe therapy for consideration as an adjunctive approach to manage cancer patient symptoms such as pain, fatigue, nausea, sleep disturbance, anxiety, loss of appetite, shortness of breath, cough and well-being. It is well tolerated by the patient and appears to provide relief in a relatively short time. Therefore, acupuncture holds great promise for alleviating suffering and preserving the dignity of patients and those who care for them.

\section{Acknowledgments}

The project was funded by the Tuscany regional health authority specifically for the purpose of healthcare and research to verify the efficacy of CAM. The authors would like to thank the administrative department at Grosseto Local Health Authority ASL 9

\section{Author Contributions}

In this paper, Franco Cracolici is the main author; he has conceived and designed the experiments, he has acquired and analyzed data and has written the research. Simonetta Bernardini has directed the research, drafted and revised manuscript; Rosaria Ferreri has collaborated in the ward, for the collection and analysis of the data with the other authors.

\section{Funding}

We declare that we have had no funding resources and grant for this research.

\section{Competing Interests}

The authors have declared that no competing interests exist. 


\section{References}

1. Clark WC, Ferrer-Brechner $\mathrm{T}$, et al. The dimension of pain: A multidimensional scaling comparison of cancer patients and healthy volunteers. Pain. 1989; 37: 23-32.

2. Mount BM. Psychological and social aspects of cancer pain. Textbook of pain. Edinburgh: Churchill Livingstone; 1984: p. 460-471.

3. Corli O. Che cos'è la medicina palliativa, in Corli O. Una medicina per chi muore. II cammino delle cure palliative in Italia. Roma: Città Nuova; 1988.

4. Parliamentary Assembly of the Council of Europe. Palliative care, a model for innovative health and social policies. 2009: 1-31.

5. Melchart D, Weidenhammer W, Streng A, Reitmayr S, Hoppe A, Ernst E, et al. Prospectivelnvestigation of adverse effects of acupuncture in 97733 Patients. Arch Intern Med. 2004; 164: 104-105.

6. Deng LY. Chinese acupuncture and moxibustion. Beijing, China: Foreign Languages Press; 1997.

7. Lu W, et al. Integrative tumor board: A case report and discussion from Dana-Farber Cancer Institute. Integr Cancer Ther. 2009; 8: 235-241.

8. Chae $\mathrm{Y}$, et al. Protein array analysis of cytokine levels on the action of acupuncture in carrageenan-induced inflammation. Neurol Res. 2007; 29: S55-58

9. Lu M, et al. Effects of acupuncture and moxibustion on DNA excision repair-related proteins of bone marrow cell in cyclophosphamide-induced mice. Zhongguo Zhen Jiu. 2009; 29: 821824.

10. Goldman N, et al, Adenosine A1 receptors mediate local anti-nociceptive effects of acupuncture. Nat Neurosci. 2010; 13: 883-888.

11. Moldenhauer $\mathrm{S}$, et al. Mobilization of CD133(+)CD34(-) cells in healthy individuals following whole-body acupuncture for spinal cord injuries. J Neurosci Res. 2010; 88: 1645-1650.

12. Alimi D, Pichard-Léandri E, Fermand-Brulé S, Dubreuil-Lemaire ML, Hill C. Analgesic effect ofauricular acupuncture for cancer pain: a randomized, blinded, controlled trial. J Clin Oncol 2003; 21: 4120-4126.

13. Hu C, et al. Acupuncture for pain management in cancer: A systematic review and metaanalysis. Evid Based Complement Alternat Med. 2016; 2016: 1720239.

14. Chiu HY, et al. Systematic review and meta-analysis of acupuncture to reduce cancer-related pain. Eur J Cancer Care (Engl). 2017; 26. doi: 10.1111/ecc.12457.

15. Molassiotis A, Bardy J, Finnegan-John J, Mackereth P, Ryder DW, Filshie J, et al. Acupuncture for cancer-related fatigue in patients with breast cancer: A pragmatic randomized controlled trial. J Clin Oncol. 2012; 30: 4470-4476.

16. Zhang Y. Effects of acupuncture on cancer-related fatigue: a meta-analysis. Support Care Cancer. 2018; 26: 415-425.

17. Bao T, Cai L, Snyder C, Betts K, Tarpinian K, Gould J, et al. Patient-reported outcomes in women with breast cancer enrolled in a dual-center, double-blind, randomized controlled trial assessing the effect of acupuncture in reducing aromatase inhibitor-induced musculoskeletal symptoms. Cancer. 2014; 120: 381-389.

18. Walker EM, Rodriguez Al, Kohn B, Ball RM, Pegg J, Pocock JR, et al. Acupuncture versus venlafaxine for the management of vasomotor symptoms in patients with hormone receptorpositive breast cancer: a randomized controlled trial. J Clin Oncol. 2010; 28: 634-640.

19. Wang $\mathrm{T}$, et al, Acupoints stimulation for anxiety and depression in cancer patients: $A$ 
quantitative synthesis of randomized controlled trials. Evid Based Complement Alternat Med. 2016; 2016: 5645632.

20. Zia FZ, et al. The National Cancer Institute's Conference on acupuncture for symptom management in oncology: State of the science, evidence, and research gaps. J Natl Cancer Inst Monogr. 2017; 1: 2017(52).

21. Garcia MK, et al. Acupuncture for symptom management in cancer care: an update. Curr Oncol Rep. 2014; 16: 418.

22. Meidell $L$, et al. Acupuncture as an optional treatment for hospice patients with xerostomia: An intervention study. Int J Palliat Nurs. 2009; 15: 12-20.

23. Deng G, Hou BL, Holodny Al, Cassileth BR. Functional magnetic resonance imaging (fMRI) changes and saliva production associated with acupuncture at LI-2 acupuncture point: a randomized controlled study. BMC Complement Altern Med. 2008; 8: 137.

24. Garcia MK, Chiang JS, Cohen L, Liu M, Palmer JL, Rosenthal DI, et al. Acupuncture for radiationinduced xerostomia in patients with cancer: A pilot study. Head Neck. 2009; 31: 1360-1368.

25. Mercadante $\mathrm{V}$, et al. Interventions for the management of radiotherapy-induced xerostomia and hyposalivation: A systematic review and meta-analysis. Oral Oncol. 2017; 66: 64-74.

26. Dean-Clower $E$, et al. Acupuncture as palliative therapy for physical symptoms and quality of life for advanced cancer patients. Integr Cancer Ther. 2010; 9: 158-167.

27. Haddad NE, Palesh O. Acupuncture in the treatment of cancer-related psychological symptoms. Integr Cancer Ther. 2014; 13: 371-385.

28. Lau $\mathrm{CH}$. Acupuncture and related therapies for symptom management in palliative cancer care: Systematic review and meta-analysis. Medicine (Baltimore). 2016; 95: e2901.

29. Bernardini S, Cracolici F, Ferreri R, Rinaldi M, Pulcri R. Integration between orthodox medicine, homeopathy and acupuncture for inpatients: Three years experience in the first hospital for Integrated Medicine in Italy. J Tradit Complement Med. 2015; 5: 234-240.

30. Bernardini $S$, et al. Effect of integrated medicine on physical performances of orthopaedic and stroke patients: A propensity score-matched study. Altern Integr Med. 2017; 6: 4.

31. Maciocia G. The foundations of Chinese medicine: A comprehensive text. Elsevier; 2017.

32. Traidou P. Les notions de physiologie et d'etiopathogenie dans la tradition medicale chinoise de la dynastie des Qing, selon le Neijing Quanshi. 1994.

33. Giullaume G. Mach-chieu: Pharmacopee et Medicine Traditionelle Chinoise, Ed. Presente; 1987.

34. Andres G. Methodologie d'etude des points d'acupuncture. 1988.

35. Larre C, Rochat de la Vallee E, Root C. Secret Treatise of the Spiritual Orchid: Huang Ti Nei Ching Su Wen, Chapter 8.

36. Di Concetto G, Sotte L, Pippa L, Muccioli M. Trattato di agopuntura e medicina cinese. UTET, Torino. 1992; 2: p. 1.268

37. Lao-Tse, Tao-Te-Ching. UTET, Torino; 1977.

38. Zanello F. Taoismo segreto: Scritti sapienziali di antichi maestri. Castelvecchi; 1993.

39. Alessandra B. Per ogni puntura, il metodo è prima di tutto di non mancare il radicamento agli Spiriti. Available from: http://www.sia-mtc.it/ cms/ pagina .asp ?id=2 23\&id_pad re=49\&tito lo_cat= Notizie.

40. Larre C, Rochat De La Vallèe E. I simboli cinesi di vita e di morte, medicina tradizionale cinese, 
il pensiero. Prima edizione italiana, Jaka Book; 2004.

41. Bedard G, et al. Minimal clinically important differences in the Edmonton symptom assessment system in patients with advanced cancer. J Pain Symptom Manage. 2013; 46: 192200.

42. Chao MT, et al. Prevalence and use of complementary health approaches among women with chronic pelvic pain in a prospective cohort study. Pain Med. 2015; 16: 328-40.

43. Mao JJ, et al. Uncovering the expectancy effect: the validation of the acupunctureexpectancy scale. Altern Ther Health Med 2010;16:22-7.

44. Malliani A. Medico sempre. Lezioni di buona sanità. Guerini e Associati; 2014.

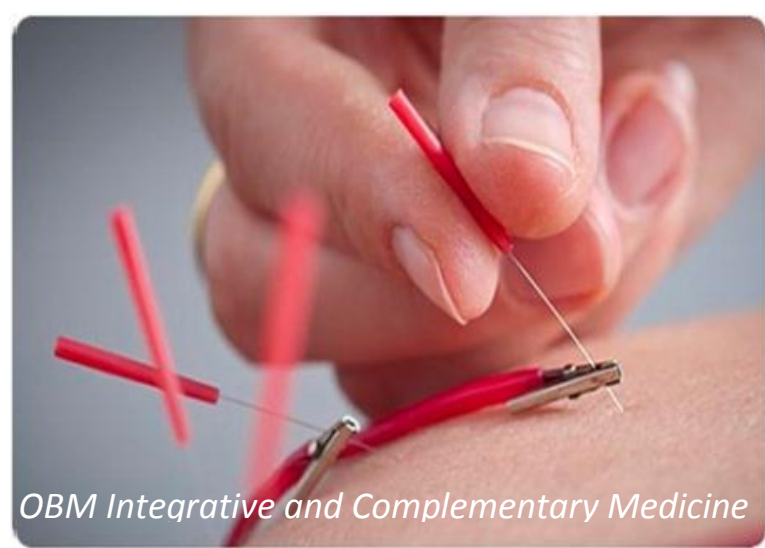

Enjoy OBM Integrative and Complementary

Medicine by:

1. Submitting a manuscript

2. Joining in volunteer reviewer bank

3. Joining Editorial Board

4. Guest editing a special issue

For more details, please visit:

http://www.lidsen.com/journals/icm 